\title{
ESTUDIOS
}

Francisco Correa Schnake

Departamento de Teología - Coquimbo

Universidad Católica del Norte

\section{Zubiri: la experiencia como vía de acceso del hombre a Dios. Una aproximación inicial a su trilogía religiosa ${ }^{(1)}$}

\section{INTRODUCCIÓN}

El tema de Dios es un problema que ha cruzado la historia de la humanidad en distintas épocas, razas y culturas. El hombre ha expresado su relación con lo divino a través de manifestaciones que en el fondo y en la forma muestran una variada gama de posibilidades y percepciones de lo que Dios ha significado para la vida del hombre.

Descontento con el tratamiento que históricamente se ha dado al tema, tanto por la teología natural como por la teodicea, Zubiri dedica grandes energías a pensar filosóficamente el problema de Dios en el contexto de la fenomenología de la religión, entendiendo por esta un pensar metafísico y riguroso.

Introducirse en la lectura de sus escritos religiosos implica enfrentarse con el tema de Dios en cuanto problema fundamental de la realidad y de la existencia humana. Dios es un problema de la realidad y, de esta forma, un problema que tiene que ver con el fundamento mismo del hombre en su hacerse persona. De allí que este problema sea problema de todos.

En este esfuerzo por pensar el tema, Zubiri crea y utiliza una serie de conceptos que le permiten expresar adecuadamente el contenido de sus intuiciones. Me limitaré aquí a señalar tres de ellos que me parecen importantes para la comprensión del tema específico que nos convoca: "teologal”, "experiencia” y "religación".

El propósito de Zubiri no es justificar el cristianismo ni ninguna otra religión, sino referirse a una situación del hombre que compete a todos como su estructura humana fundamental, es decir, en cuanto "experiencia teologal". El problema de la divinidad es algo que se nos plantea por el solo hecho de ser hombres, situación que da cuenta de una dimensión constitutiva y estructural de la propia realidad humana, y en cuanto tal es previa a cualquier intento de discurso teológico sobre Dios. A esto se refiere con lo de "teologal". Esta dimensión estructural y constitutiva del hombre

(1) Aprovecho la ocasión de agradecer a ICALA, institución que con su aporte de una beca hizo posible la realización de este estudio. 
es la que lo impulsa y obliga, quiera o no, a buscar el fundamento último de la realidad, lo obliga a la realidad y a Dios como lo ineludible.

En este contexto, es importante el rol que Zubiri asigna al tema de la "experiencia" (2) como vía de acceso y, fundamentalmente, como posibilidad de encuentro del hombre con Dios. Afirma explícitamente, en la tercera parte de su obra $E l$ Hombre y Dios, que el hombre es experiencia de Dios y que Dios es experiencia del hombre en una relación de "unidad experiencial", en cuanto transcurso de la experiencia fundamental del hombre como "religación". La unidad experiencial del hombre y Dios apunta a una relación primaria fundamental de "probación" de la realidad y no de comprobación teórica de esta.

La "religación" es la experiencia de estar fundados en la realidad y remitidos estructuralmente a ella y en esa medida es experiencia, probación de la condición de religación del hombre a la realidad. Ambos conceptos son claves para entender el pensamiento del autor sobre la "experiencia teologal" del hombre y, en él, específicamente el tema de la "experiencia en cuanto vía de acceso del hombre a Dios". A su profundización nos abocaremos en la segunda parte del artículo.

En la elaboración de este trabajo como acercamiento inicial a la obra del autor, me pareció oportuno tener presente dos criterios fundamentales que permiten una adecuada comprensión del tema objeto de este artículo: por un lado, seguir de cerca el planteamiento que el autor hace del tema en su libro El Hombre y Dios (3) con una mirada especial en la tercera parte de la obra y, por otro, en forma complementaria, una mirada general transversal del tema en su trilogía religiosa.

El artículo está estructurado en tres partes. En la primera, se señala una contextualización del tema en el conjunto de la obra religiosa de Zubiri. En la segunda, se desarrolla el tema de la experiencia en cuanto vía de acceso del hombre a Dios. Finalmente, se ofrece una breve síntesis de los elementos más significativos que han ido surgiendo en el transcurso del trabajo (4).

(2) Cf. "Experiencia", en: Ferrater Mora, Diccionario de Filosofía, Barcelona 1999, 1181-1188. El rol fundamental que Zubiri asigna a la "experiencia" entendida como probación de la realidad dista mucho de otras concepciones con que históricamente se ha entendido este concepto. Una breve mención hacemos a esta situación en la segunda parte del trabajo.

(3) Obra en que desarrolla los aspectos fundamentales de su pensamiento sobre el tema.

(4) Antes de introducirse en la lectura del texto me parece oportuno informar al lector acerca de la dificultad que presenta el tema de la autoría final de Zubiri respecto de los escritos en que aborda el problema de Dios. Para una adecuada comprensión de este problema es conveniente consultar la presentación que se hace a cada una de sus obras, ya que estos textos vieron la luz después de su muerte y poseen distintos niveles de trabajo y preparación final por su autor.

A partir de esta situación es posible establecer dos criterios importantes en relación con la manera de acceder a los textos de la trilogía religiosa: el primero de ellos tiene que ver con la necesidad de establecer como criterio básico de lectura de los textos póstumos de Zubiri los planteamientos desarrollados en su trilogía sobre la inteligencia, por considerar que en esta obra estaría el pensamiento maduro y definitivo del autor. Como representante de esta postura puede verse Diego Gracia, Voluntad de Verdad, Barcelona 1986, prólogo pg. VII (en adelante se citará de la siguiente manera: D. Gracia, Voluntad). "Más aún, pienso que los inéditos deberán estudiarse desde los escritos de los últimos años, en particular desde el tríptico de la inteligencia, y no al revés". El segundo criterio apunta a la necesidad de leer sus textos póstumos desde sí mismos, sin criterios previos de interpretación, ya que esta sería la única posibilidad de encontrarse con lo que el autor realmente ha querido afirmar. Como representante de esta preocupación puede verse. Andrés Torres Queiruga, El hombre como experiencia de Dios en Zubiri, en: Ángel Álvarez Gómez y otros, la filosofía de Zubiri en el contexto de la crisis europea, Santiago de Compostela 1996, 167-185 (en adelante se citará como A. Torres, El hombre). 


\section{CONTEXTUALIZACIÓN Y APORTE DE XAVIER ZUBIRI AL TEMA DE LA EXPERIENCIA COMO VÍA DE ACCESO DEL HOMBRE A DIOS}

\section{a) Importancia del problema de Dios en la obra de Zubiri}

Tres son los problemas, que según el propio Zubiri, lo han desafiado toda la vida: el problema de la realidad (5), el problema de la inteligencia (6) y el problema de Dios (7).

Para el autor el tema de Dios debe ser abordado como un verdadero problema, no como un problema más sino como el que tiene directa relación con el mismo fundamento del hombre en su hacerse persona y, en este sentido, como un problema de todos y de todo, tenga el hombre conciencia de ello o no (8).

Frente a este problema siempre han existido diversas respuestas y soluciones. Algunas de carácter positivo y otras, negativo. Positivamente responde el creyente y negativamente quien se declara ateo o agnóstico, guardando las distinciones en sus planteamientos. Lo importante de todas estas respuestas es que pueden olvidar que son soluciones a un problema real, el problema de Dios (9). El ateo puede pensar que Dios no es su problema ya que no es creyente y no afirma su existencia, por lo que, desde su perspectiva, el problema es del creyente. El creyente puede pensar que Dios no es su problema porque reconoce su existencia y cree en él (10), entendiendo que existen distintos conceptos de fe, y, por lo tanto, quien tiene un problema, según su percepción, es el ateo ya que es él quien debe dar razones para negar la existencia de Dios. La dificultad, en ambos casos, radica en no comprender que sus soluciones lo son, en primer término, de un problema real.

Hoy en día, la situación de un amplio margen de hombres se caracteriza más bien por una actitud de mayor radicalidad frente al tema. Lo que se niega no es si Dios existe o no, sino la existencia de un verdadero problema de Dios. Tanto creyentes como no creyentes niegan tener un problema. En esta línea, el esfuerzo esencial de Zubiri es demostrar que Dios sí es un problema central para todos, independientemente de la respuesta que frente a él se pueda dar. Tanto el creyente como el no creyente, sea este ateo o agnóstico, deben dar las razones de la solución por la que han optado, es decir, deben saber fundamentar las opciones de su vida.

Para Zubiri, Dios no es un problema que el hombre pueda o no plantearse, como si se tratara de un problema cualquiera que demanda ser abordado como algo

(5) Cf. Sobre la Esencia, Madrid 1962.

(6) Cf. su trilogía sobre la inteligencia, publicada entre los años 1980 y 1983: Inteligencia Sentiente, Madrid 1980. Inteligencia y Logos, Madrid 1982. Inteligencia y Razón, Madrid 1983.

(7) Cf. El hombre y Dios, Madrid 1998 (en adelante se citará: HD). Al morir Zubiri deja inconcluso este libro, que posteriormente será editado bajo la dirección de su discípulo Ignacio Ellacuría en Madrid en 1984. Los otros dos textos que componen la trilogía religiosa de su obra son publicaciones póstumas de la década de los noventa.

(8) Cf. Moisés González Moreno, Dios problema de todos. Zubiri y el problema de Dios, en: http/ servicioskoinonia.org/relat/; María Lucrecia Rovaletti, La dimensión Teologal del Hombre. Apuntes en torno al tema de la religación en Xavier Zubiri, Bs. As. 1979, 9. La autora se atreve a plantear que el tema de Dios habría sido, dentro de los tres temas que preocuparon a Zubiri, el motor fundamental de su pensamiento.

(9) Cf. HD, 369-383.

(10) Cf. Ibíd, 209-304. En que desarrolla el tema de la fe en cuanto raíz formal del acceso del hombre a Dios. 
externo. El problema de Dios es un problema que el hombre debe plantearse, quiéralo o no, sépalo o no, ya que obedece a su misma condición de hombre. Es decir, pertenece a la realidad humana en cuanto tal y en este sentido es ineludible. Zubiri se refiere a él como una cuestión teologal y no teológica en primer término. Teologal en cuanto se refiere a una realidad humana que envuelve formal y constitutivamente el problema de Dios y, en tal sentido, es una cuestión previa a la teología. Lo teologal se conforma en una estructura humana que es accesible a un análisis inmediato. De tal manera, que Dios es estrictamente un problema que el hombre debe abordar seriamente en cuanto expresión fundamental de su propia problematicidad y estructura humana. Al respecto afirma: "Lo teologal es, pues, en este sentido, una estricta dimensión humana, accesible a un análisis inmediato. A ella hemos de atender. La puesta en claro de esta dimensión es la mostración in actu exercito de la existencia del problema de Dios, en tanto que problema. El problema de Dios, en tanto que problema, no es un problema cualquiera, arbitrariamente planteado por la curiosidad humana, sino que es la realidad humana misma en su constitutivo problematismo" (11).

El aporte de Zubiri al tema del acceso del hombre a Dios consiste básicamente en señalar que tal acceso supone necesariamente la experiencia humana. Este es el camino que posibilita el encuentro con la realidad fundante del hombre, es decir, con Dios. Este esfuerzo implicará necesariamente un replanteamiento del concepto de experiencia.

\section{b) Un esfuerzo por asumir y plantear situadamente el tema de Dios}

Un segundo elemento a considerar tiene relación con el esfuerzo por pensar e intentar expresar a Dios y la religión situadamente (12), es decir, con un lenguaje y argumentos razonables para el hombre de hoy. Zubiri entiende que en el pasado se intentó acceder a Dios por vías que hoy son consideradas claramente impracticables. Una de ellas fue la realizada por la "teología natural" -cuyo inicio podría remontarse a Grecia llegando hasta la Edad Media-, que consideraba posible probar la existencia de Dios partiendo de la experiencia sensible y utilizando recursos como el orden del universo o el principio de causalidad, es decir, las llamadas pruebas cosmológicas, cuya expresión paradigmática fueron las cinco vías de Santo Tomás de Aquino. Zubiri piensa que este intento es una vía muerta para el hombre de hoy (13).

Frente a esta visión surge como alternativa la propuesta del racionalismo moderno que funda la "teodicea", basada en el argumento ontológico, la cual sostiene que a Dios no se puede llegar por la naturaleza sino por la razón pura. Se produce el reemplazo de las antiguas pruebas cosmológicas por el argumento ontológico. Al respecto Zubiri entiende que también resulta necesario superar esta visión, pues culmina en un idealismo que es igualmente vía muerta.

(11) Cf. Ibíd, 372.

(12) Cf. Diego Gracia, Zubiri y la Experiencia Teologal. La difícil tarea de pensar a Dios y la religión a la altura del siglo XX, en: http/www.Zubiri.net/index $2 \mathrm{html}$. Foro, textos a debate, el problema teologal (Diego Gracia). (En Adelante se citará: D. Gracia, Zubiri).

(13) Cf. HD, 118-123. 
Una tercera vía, conocida bajo el nombre de "filosofía de la religión", comienza a gestarse a mediados del siglo XVIII. Ya no se intentará llegar a Dios por la vía de la lógica, a través de juicios sintéticos o analíticos, como sucede en la teología natural o en la teodicea respectivamente. El fenómeno religioso es percibido como una realidad mucho más compleja que no permite ser reducida fácilmente a esquemas lógicos. El desafío está en pensar a Dios y la religión después de la crisis del racionalismo.

Esta tercera vía, en la que no han estado ausentes las dificultades, recibió un fuerte impulso del movimiento conocido bajo el nombre de "fenomenología de la religión" (14). Es en este esfuerzo de la fenomenología de la religión, entendida como un pensar riguroso y radical, es decir metafísico, en el que es preciso situar el aporte de Zubiri al tema que nos ocupa (15).

Para él es evidente que el hombre no puede llegar a Dios, si él previamente no se le hace presente. De ahí que a Dios se le encuentre, más que por la vía de la razón analítica o sintética, por la vía de la experiencia, entendiendo que la experiencia junto con el esbozo son dimensiones de la razón. Su intención no es negar la razón sino entenderla en un sentido más amplio, capaz de integrar la experiencia como probación de la realidad esbozada.

La noción de experiencia de Dios aparece como la gran intuición de Zubiri en relación al problema del acceso del hombre a Dios. Intuición que desarrollará desde diferentes perspectivas en su trilogía sobre el "problema teologal del hombre". En El Hombre y Dios trata de la vida humana en cuanto experiencia constante de Dios en cuanto fundamento; en El problema filosófico de la historia de las religiones (16) analiza la experiencia de Dios en la historia de las religiones; y en El problema teologal del hombre: Cristianismo (17) desarrolla el esfuerzo por identificar y definir lo propio de la experiencia cristiana entendiéndola como la máxima manifestación de Dios al hombre, experiencia de la encarnación, en que se da la experiencia de la "deiformidad" y de la "deificación".

\section{c) Trasfondo filosófico-teológico}

Una adecuada comprensión del pensamiento del autor implica necesariamente su contextualización.

Desde la perspectiva filosófica es claro el influjo que sobre él tuvieron Ortega y Heidegger (18). De igual modo, desde la perspectiva teológica se aprecia con

(14) Cf. D. Gracia, Voluntad, 33-78.

(15) Cf. D. Gracia, Zubiri, 2. "Si hubiera que situar el esfuerzo de Zubiri en un contexto concreto, este no podría ser otro que el de la fenomenología de la religión. En su base están los nombres de Nathan Söderblom, Rudolf Otto, Friedrich Heiler, Martín Heidegger. Zubiri va a partir de estos materiales, y desde ellos se va a proponer, en el tema de Dios, la misma tarea que en las otras áreas de la filosofía y la metafísica, radicalizar el método fenomenológico, llevarlo hasta sus últimas consecuencias, y a partir de ahí elaborar todo el edificio de la metafísica".

Junto con ello es interesante el texto de A. Torres, El hombre, 174 ss. El autor expresa con claridad la dependencia de Zubiri de algunos planteamiento de K. Rahner y posiblemente también de Hegel.

(16) Madrid 1993.

(17) Madrid 1997.

(18) Cf. D. Gracia, Voluntad, 33-78. 
claridad el influjo del ambiente teológico renovador del pre y post Concilio, básicamente a través de Karl Rahner (19), en concreto en temas como el "existencial sobrenatural", la "causalidad quasi-formal" de Dios en el hombre, de Dios en cuanto "constitutivo formal" de todo, de la "trascendencia en el mundo", del hombre como Dios ad extra, del "cristianismo en tanteo" (20) y otros.

En esta perspectiva, el esfuerzo de Zubiri puede sintetizarse básicamente en dos intenciones fundamentales: en primer lugar, la de asumir el tema de Dios que no había sido trabajado directamente por la filosofía contemporánea, expresándolo a través de un lenguaje y perspectivas propios y, en segundo lugar, realizar este esfuerzo desde una dialéctica que sabe volver críticamente a las intuiciones fundamentales de la filosofía occidental sobre el tema, repensándolas y exponiéndolas adecuadamente para ser entendidas por los hombres de nuestro tiempo.

\section{d) Experiencia de Dios, un tema siempre abierto}

El pensamiento de Zubiri, quien gozó de una larga vida filosófica, es reflejo de un esfuerzo permanente de construcción y reelaboración. Esta sensación de constante búsqueda se hace más fuerte en su tratamiento del problema de Dios (21), un tema que lo acompañó a lo largo de toda su vida y que tras su muerte ha seguido abierto en sus grandes intuiciones. Por lo mismo, es razonable pensar que Zubiri no pretende decir la última palabra o dar por terminado el tema de la experiencia de Dios con el desarrollo de sus ideas. Más bien, da la impresión de una lúcida conciencia de estar frente a una cuestión extremadamente difícil de abordar, en la que se puede avanzar pero en la que difícilmente existe una palabra definitiva. Esto se debe a que la experiencia de Dios se realiza en la experiencia humana más radical, en aquella en que surgen las preguntas finales que nunca alcanzan una respuesta definitivamente adecuada. Frente a ello, el mayor peligro del que es preciso cuidarse consiste en la tentación de reducir la experiencia de Dios a una comprensión previa y particular que, por lo mismo, no puede ser de Dios. Frente a esta tentación tan humana, Zubiri realiza un valioso aporte.

\section{RELIGACIÓN Y EXPERIENCIA: "EXPERIENCIA DE RELIGACIÓN"}

\section{a) Contextualización del tema de la experiencia}

Sin pretender realizar un análisis completo y acabado de lo que en la obra del autor se entiende por experiencia, por cuanto ello implicaría un esfuerzo que supera las posibilidades de este artículo, nos centraremos en su libro El Hombre y Dios en el que desarrolla algunos intentos de explicación de su concepto de experiencia.

(19) Cf. Xavier Zubiri, El Problema Teologal del Hombre, en: A. Vargas-Machuca (ed), Teología y mundo contemporáneo. Homenaje a Karl Rahner, Madrid 1975, 55-64. Y reproducido en HD 365-383.

(20) Cf. A. Torres, El hombre, 174-175. Conviene leer también las notas № 32-34 de este mismo trabajo en que se explicitan los nexos con el desarrollo teológico de Karl Rahner en estos puntos. Cf. HD, i-x (presentación de Ignacio Ellacuría). 
Para ello será necesario hacer previamente una breve descripción general de lo que entiende por religación, ya que solo en este contexto el concepto de experiencia que utiliza es comprensible.

La religación a la que Zubiri se refiere en cuanto eje articulador de su pensamiento (22), no tiene nada que ver con una comprensión tipo vía de causalidad o razón necesaria y suficiente, sino que tiene relación con el análisis y la descripción de una experiencia fundamental, de un dato primario, en la profundización de la existencia humana en cuanto experiencia "yectiva" (23). Pero la intuición de este carácter fundamental no basta, según Zubiri; es preciso profundizar esta experiencia entendiendo que se trata de un estar "yecto desde", es decir, "puesto por atrás o desde atrás", lo que indica que la iniciativa, respecto de la religación, no la tiene en primer término el hombre sino que le viene dada de fuera (24).

El hombre está religado a la realidad mundana, la única que le es accesible. Esta realidad, a la que el hombre se encuentra religado, se apodera del hombre y se le impone (25). No es el hombre el que se apodera de la realidad sino que es esta la que se apodera de los hombres. A esto se refiere Zubiri cuando habla del poder de lo real, con sus notas características de "ultimidad", "posibilitancia" e "impelencia", siendo esta última la que incita al hombre a ser y a realizarse en la búsqueda del "fundamento" (26) de la realidad. Fundamento que se impone realmente y, por ello, adopta la forma de "poder" que se "impone" y "religa" de manera intraaprehensiva. Pero no solo religa, sino que también y al mismo tiempo, lanza "hacia" el allende, en una búsqueda de "intelección racional" del fundamento allende para comprender lo que puedan ser las cosas en la realidad del mundo. No se trata, por tanto, de un mero razonamiento o principio lógico, sino de un verdadero poder real que se experimenta en el mundo (27).

(22) Cf. A. Torres, El Hombre, 168-170.

(23) Cf. D. Gracia, Zubiri, 4-5, para quien obviamente Zubiri está siguiendo los análisis heideggerianos de la Geworfenheit, es decir, el estar yectado o arrojado. Diego Gracia reconoce un aporte zubiriano sobre el tema en el intento de ir más allá de su formulación profundizando en este carácter.

(24) Ibíd, 5. Respecto de este tema, afirma: "Zubiri utiliza, para explicar esto, la diferencia entre la vis a fronte y la vis a tergo. La proyección, el proyecto nos lanza siempre hacia delante, es una vis a fronte, en tanto que la religación es una vis a tergo que nos liga por detrás. Las dos vis ligan, pero de modo distinto. La vis a fronte es para él el fundamento de la "obligación", en tanto que la vis a tergo lo es de la "religación". Es la diferencia que media entre la ética y la religión. ... Para Zubiri la obligación no es un fenómeno originario, sino que siempre se halla fundado en algo anterior a ella misma, que es el dato primario de la religación (cf, p. e., Vol. 1, p. 93; Vol. 3, p. 306)".

(25) Cf. Xavier Zubiri, Naturaleza, Hombre, Dios, Madrid 1987, 373. "La existencia humana, pues, no solamente está arrojada entre las cosas, sino religada por su raíz”. (En adelante se citará: NHD).

(26) Cf. D. Gracia, Zubiri, 5. Nuevamente nos encontraríamos en presencia de un concepto fundamental en el pensamiento de Zubiri que nos remitiría a Heidegger y a su obra de 1929 titulada Vom Wesen des Grundes (sobre la esencia del fundamento): "En ese trabajo Heidegger se distancia de cualquier interpretación del fundamento como "causa" (al modo del realismo antiguo) o como "principio" (al modo racionalista moderno). En los dos casos se parte de la "posición" de la mente, en tanto que el fundamento de que habla Heidegger no viene puesto sino "ofrecido"; es una oferta, un don, un regalo. Pues bien, en Zubiri sucede algo similar".

(27) Cf. Ibíd, 6, la aclaración de Diego Gracia en relación al tipo de razón a la que se está refiriendo Zubiri. Al respecto, afirma: "La razón de Zubiri ya no es la del racionalismo. La razón, lejos de resolver los problemas, lo que hace más bien es plantearlos. La razón es 'problemática'. Y por eso consiste en búsqueda. Es, ciertamente, búsqueda 'racional', pero continua e interminable, precisamente porque no puede acabar completamente con los problemas. Puede intentar resolverlos, pero desde luego no disolverlos". 
Zubiri entiende que la razón consiste básicamente en ser "método", siempre en camino y dinamismo, que consta de dos pasos fundamentales: el "esbozo" y la "experiencia" (28). En el caso de Dios, el esbozo, argumento siempre lógico, tiene directa relación con la religación y consiste en la formulación de un argumento sobre Dios. Reconociendo el valor del argumento, que no es prueba y, por lo tanto, no tiene la capacidad de llevar al hombre al encuentro con Dios, se requiere del segundo paso del método que es la razón, el momento de la "experiencia" que básicamente es momento de "conformación” y "compenetración”. Aquí sí estamos en presencia de la prueba, pero no en sentido lógico, sino experiencial o físico. Al respecto es interesante la siguiente afirmación de Diego Gracia:

“Así define Zubiri la experiencia, como 'probación física de la realidad'. De lo que resulta que la prueba de Zubiri, si es que se quiere hablar de prueba, no es racional o lógica sino experiencial o física. Una vez elaborado el esbozo sobre Dios, el ser humano tiene que probarlo realmente en su vida, bajo modo de conformación y compenetración. Es la experiencia de Dios. Se trata, de algún modo, de una versión nueva de la célebre 'apuesta' pascaliana. Y por supuesto también de la versión zubiriana de lo que Heidegger denomina con el término Ereignis" (29).

Zubiri entiende que no siempre la razón puede dar el salto desde la religación, desde el poder de lo real, a la religión, realidad personal de Dios, y esto se explicaría a partir de la posibilidad de error en el argumento o en la falta de la experiencia. Ahora bien, cuando el salto sí se produce, el poder de lo real cobra figura personal en Dios y las propiedades del poder de lo real; "ultimidad", "posibilitancia" e "impelencia", se constituyen en manifestaciones de Dios en el mundo, entendidas como "acatamiento", "súplica" y "refugio".

\section{b) Intento de delimitación del concepto de experiencia en el ámbito de la religación}

Zubiri indica directamente que cuando habla de experiencia, en El Hombre y Dios, no se refiere al dato sensible, ni al reconocimiento de una misma cosa en distintas percepciones, ya que experiencia no sería lo mismo que lo empírico. Tampoco lo utiliza en el sentido de la expresión experiencia de vida (30). A lo que se refiere cuando habla de experiencia es a una prueba, que no tiene carácter de com-

(28) Cf. D. Gracia, Voluntad, 216. “La religación tiene, pues, un carácter experiencial (HD 95). ... La religación 'da que pensar', y obliga al hombre a poner en marcha el 'método racional'. Este, como también sabemos, parte de un 'sistema de referencias', que es siempre la cosa en tanto que actualizada en la aprehensión, y que el tema de Dios es precisamente la religación. A partir de este sistema de referencias se construye un 'esbozo' de lo que podría ser la cosa -en este caso Dios- en la realidad. Y luego se retorna a la realidad, para verificar si ella aprueba o reprueba el esbozo: es el tercer momento del método racional, la 'experiencia', entendida como probación física de la realidad". Para mayor detalle del análisis que hace nuestro autor sobre el tema puede consultarse: X. Zubiri, Inteligencia y razón, Madrid 1983, 202-257.

(29) D. Gracia, Zubiri, 6.

(30) Cf. HD, 95. "Experiencia no significa aquí la aísthesis, es decir, no es el dato sensible. Tampoco es lo que Aristóteles llamó empeiría, el reconocimiento (mnéme) de una misma cosa en distintas percepciones; experiencia no es aquí lo empírico. Tampoco significa lo que designamos como experiencia de vida". 
probación, ni conceptual, ni de ningún otro tipo, sino más bien "probación” de la realidad física de algo (31).

"Una especie de prueba a que se somete algo, una prueba que no es mera comprobación, por ejemplo conceptiva, sino que es el ejercicio mismo operativo del dato de probar: es probación física. ¿De qué? De la realidad de algo. La experiencia es, pues, probación física de realidad" (32).

Se trata básicamente de la experiencia de la religación al fundamento de la propia existencia humana: "El hombre se dirige a la realidad para buscar un apoyo en ella, y a su vez esta realidad tiene gran riqueza de notas, las cuales son una talificación del momento de realidad, y por tanto quedan determinadas por este momento como posibilidades de realización. La inserción de estas posibilidades en la realización de mi persona es la probación física de realidad. El hombre haciendo religadamente su propia persona, está haciendo la probación física de lo que es el poder de lo real. Es la probación de la inserción de la ultimidad, de la posibilitación y de la impelencia en mi propia realidad. Al hacerme realidad personal soy pues una experiencia del poder de lo real, y por tanto de 'la' realidad misma. Esta probación se va ejercitando por todas las rutas individuales, sociales y históricas” (33) (sic).

\section{c) El hombre y la religación: experiencia de Dios}

Para Xavier Zubiri es claro que Dios se encuentra en el término de la religación personal. A Dios se le descubre en el análisis de la existencia humana (34). Dar adecuada cuenta del acontecimiento de la religación del hombre y de su finalización en Dios requiere de una breve síntesis de los elementos trabajados a lo largo del texto El Hombre y Dios.

Sin pretensión de exhaustividad, sintetizaré los elementos que permiten abordar de mejor forma el tema de la religación del hombre en cuanto experiencia de Dios.

\section{c.1. Breve contextualización}

El hombre en su religación está religado precisa y formalmente en su ser personal al "poder de lo real", en el cual y desde el cual hace y se hace (35).

(31) Esto no implica que esté del todo claro y definido el tema de la experiencia en Zubiri, tal como lo plantean A. Torres, El hombre, 170-171, cuando aborda el tema de la religación como radicación experiencial; Jesús Sáez Cruz, La accesibilidad de Dios: su mundanidad y transcendencia en X. Zubiri”. Salamanca 1995, 263. En la nota № 20: “Zubiri, aunque en su obra 'Hombre y Dios' describe la experiencia como 'probación de realidad' con mucha frecuencia..., en algún momento pudiera parecer que todavía no ha llegado a asimilar en HD lo que esta expresión significa en IRA 222-257. Como, por ejemplo, cuando entiende por experiencia la 'versión' (HD 317) que es el animal de realidades. Independientemente de que en 'Inteligencia y Razón' haya profundizado sobre el concepto de experiencia, la equiparación entre 'animalidad' y 'experiencia' alude simplemente al carácter sentiente de la versión a la realidad y de la experiencia misma de Dios".

(32) HD, 95.

(33) Loc. cit.

(34) Cf. NHD, 367: "La cuestión acerca de Dios se retrotrae así a una cuestión acerca del hombre. Y la posibilidad filosófica del problema de Dios consistirá en descubrir la dimensión humana dentro de la cual esa cuestión ha de plantearse, mejor dicho, está ya planteada".

(35) CF. HD, 99-112. 
Este poder de lo real está "en" las cosas reales y por ello, la religación es una experiencia manifestativa del poder de lo real y manifestación de lo real en sí mismo, en y por las cosas reales. Refiriéndose a la religación Zubiri expresa: "es experiencia en cuanto probación física de la realidad, pero que es también experiencia manifestativa y ostensiva en alguna manera de ese poder de lo real, y de la realidad que tiene ese poder. En mi religación, pues, se va a manifestar en experiencia lo que es la realidad y el poder de lo real. Vamos aprehendiendo en y por la realidad misma qué es eso de la realidad" (36).

Este poder de lo real, en el cual el hombre hace y se hace que está presente en las cosas reales, es real en cuanto está fundado en una "realidad fundamento". Al respecto afirma: "La persona humana, por la estructura misma del poder de lo real al que se encuentra religada, se halla, en efecto, remitida a una realidad-fundamento, esto es, a una realidad absolutamente absoluta" (37).

Este poder es la trascendencia de Dios en las cosas. Dios no está allende las cosas mismas, ya que es algo mucho más fundamental y anterior a cualquier teoría. "Es Dios transcendente en las cosas: no es transcendente $a$ las cosas, sino transcendente en las cosas. Y entre ellas transcendente en la persona humana" (38).

La religación en cuanto momento fundamental de la realidad personal implica que la realidad de Dios es, en tanto realidad, absolutamente absoluta y, por lo mismo, radicalmente distinta de todas las cosas reales y realidades personales, y está, al mismo tiempo, presente en toda realidad personal y humana en cuanto esta se hace. Este carácter unitario y trascendente, esta manera de relación que se da en la religación, que no debe entenderse en forma aditiva o copulativa de partes distintas, es expresado a través del carácter de fundamento que tiene la realidad en cuanto realidad fundamento fundamentante de todo lo real (39). En este sentido, la religación es una "presencia de Dios en las cosas, constituyéndolas formalmente, constituyéndolas como realidades. Y en el caso de las personas humanas, es algo que está constituyendo mi remisión al fundamento divino de mi propia realidad personal en la configuración de mi propio Yo. Es una realidad que está justamente fundando. Fundantemente es como está Dios en las cosas y en el fondo de las personas más especialmente" (40).

Este carácter especial le viene al hombre de su condición de "esencia abierta" (41) que como tal se relaciona de manera distinta y particular con Dios respecto de

(36) Ibíd, 307-308.

(37) Ibíd, 308.

(38) Loc. cit.

(39) Cf. Ibíd, 309.

(40) Loc. cit.

(41) Cf. Ibíd, 314-15. Zubiri entiende que existen dos tipos fundamentales de realidades metafísicas: esencias cerradas (las cosas) y abiertas (personas) que por sus propias características suponen maneras diferentes de relaciones con su fundamento. Refiriéndose a ellas sostiene respecto de las esencias cerradas: "Son cosas que tienen sus propiedades, que son las propiedades de su realidad en tanto que realidad, y actúan conforme a ellas. En ese caso el modo de fontanalidad consiste en que Dios es la fuente del ser "de suyo" lo que las cosas son". Y refiriendo a las esencias abiertas se trata fundamentalmente de aquellas que siendo 'de suyo' son 'suyas'. Al respecto indica: "Se comportan con su propio carácter de realidad, cosa que no les pasa al resto de las realidades cósmicas. Son las esencias abiertas, abiertas a su propio carácter de realidad. Es el caso del hombre. ... El hombre actúa por ser y para ser una forma de realidad, es decir, con vistas a su propio carácter de realidad". 
las "esencias cerradas". La relación entre Dios, "realidad absolutamente absoluta fundamentante" (42), y el hombre, "realidad relativamente absoluta fundamentada" (43), es expresada por Zubiri en la siguiente afirmación: "Dicho in genere, Dios me está presente, me está fundamentando en mi religación, que es un momento de mi propio Yo; ... Justo cuando y en la medida en que el poder de lo real es algo que en religación vamos a experienciar. Por tanto, Dios y la persona humana se encuentran incursos, van incursos en esta experiencia de lo real. Como la religación es un momento formalmente constitutivo de mi persona, resulta que Dios y la persona están determinados en y por esa experiencia de Dios, por lo menos en tanto que fundamentante. El hombre, dicho en términos de tesis, es experiencia de Dios. He aquí la radical unidad de Dios y de la persona humana. Es el momento de la ' $y$ '. ' $\mathrm{Y}$ ' es por lo pronto ser el hombre experiencia de Dios" (44).

Afirmar que el hombre es experiencia de Dios (45) supone comprender que el hombre, en su propia realidad personal, está "experienciando" la realidad de Dios (46). Dios no se suma al hombre como algo externo a lo que este tiene que llegar, por el contrario, le afecta y le constituye en su núcleo más íntimo. Solo así puede comprenderse que la relación con Dios esté planteada fundamentalmente como experiencia.

Al respecto resultan muy iluminadoras las palabras de Torres Queiruga: "Se trata de caer en la cuenta de que Dios está ya en la existencia cuando el hombre se interroga sobre sí mismo o se pregunta por Dios. De modo que ser sujeto consiste formalmente en estar no solo abierto a Dios, sino en estar siendo y viviendo desde él. Hasta el punto de que, cada vez que el hombre se pregunta por sí mismo, está necesariamente, quiéralo o no, sépalo o no, preguntando también por Dios" (47).

(42) Cf. Ibíd, 312-313. Zubiri afirma que "Dios está en toda realidad, en toda cosa, en cuanto realidad fundante. A la realidad pertenecería intrínsecamente el ser dinámica, es decir, el 'dar-de-sí'. Si en último término la realidad es Dios y lo es de modo fundante, se entiende que Dios 'dé-de-sí' y no pueda no dar, ya que lo propio de sí sería justamente 'dar-de-sí'. Dios es un 'dar-de-sí' de la realidad misma en que él consiste 'y que precisamente por ser algo que da-de-sí la realidad, en tanto que realidad de lo que no es Dios, precisamente por eso es fundante de toda esta realidad"." Este "dar-de-sí" de lo real, en tanto que real, consiste precisamente en la trascendencia de Dios en aquello que "da-de-sí", es decir, lo real. En este sentido, Dios consiste, desde el punto de vista de la fundamentación, en ser el fondo último y radical en el que emerge y en el que está la realidad del hombre en tanto que real. Al respecto afirma: “...en la medida en que Dios está constituyendo in actu exercito y en todo instante ese carácter de realidad por su transcendencia en las cosas, Dios es algo que está presente en el fondo de todas ellas de una manera continua, constante y constitutiva".

(43) Cf. Ibíd, 315. Zubiri entiende que existe un carácter especial de fontanalidad en las esencias abiertas, ya que en este caso no se trata de un dar meramente fontanal, sino de un dar más íntimo: es donación de sí mismo.

(44) Ibíd, 309-310.

(45) Zubiri realiza el tratamiento del tema de la experiencia unitaria de Dios y el hombre desde diferentes perspectivas. Sin desconocer esta situación y en razón de la orientación de este trabajo me centraré exclusivamente en el desarrollo de sus intuiciones respecto del "hombre en cuanto experiencia de Dios".

(46) Cf. HD, 315. Se trata de una relación interpersonal en que de alguna forma, la realidad de Dios constituye la realidad humana (esencia abierta). La donación de Dios al hombre tiene un carácter especial ya que en ella Dios le da a la persona humana su verdad real. Respecto del tema de la "verdad real" puede consultarse HD, 316-324.

(47) A. Torres, El hombre, 173. 


\section{c.2. El hombre, experiencia de Dios}

Entender correctamente lo que se quiere afirmar al decir que "el hombre es experiencia de Dios", implica comprender que en primer término y formalmente el hombre "no tiene" una experiencia de Dios, sino que primera y formalmente "es" experiencia de Dios (48). Se trata de una experiencia que es el hombre y no de una mera relación con un objeto llamado Dios, ni tampoco de un estado en el que el hombre estaría.

\section{c.2.1. El hombre, experiencia de finitud}

Para Zubiri, el hombre es una manera finita de ser real y efectivamente Dios. La naturaleza humana es ese momento de finitud, múltiple y variado, que en el caso del hombre muestra una estructura determinada. El hombre "es una manera finita de ser Dios" (49). Y esta finitud es formalmente experiencia.

El hombre es un animal de realidades y esa condición de animalidad que es suya, en cuanto esencia abierta, es la manera como está incluido su modo experiencial. En este sentido, el hombre es un modo experiencial de ser Dios, realidad absolutamente absoluta.

Frente a Dios el hombre es una realidad relativamente absoluta. Relativa debido a que este carácter absoluto lo tiene cobrado frente a la realidad haciéndose persona, haciendo y fabricando su propio yo, en una marcha que es en rigor "tanteo" (50).

Dios es aquello que está fundando y haciendo posible lo absoluto de mi ser. "La experiencia de lo absoluto no es otra cosa sino la experiencia de lo absoluto cobrado en la constitución de mi ser, la experiencia de estar fundado en una realidad fundante" (51). Dios está inscrito en la relatividad, en la persona relativamente absoluta, en la propia realidad personal y, por lo mismo, existen distintos modos de estar presente. La propia experiencia de la relatividad absoluta del hombre es justamente la experiencia de Dios.

\section{c.2.2. Modos o niveles de la experiencia de Dios}

\section{c.2.2.1. Experiencia universal de la religación, propia de todo ser humano:}

Este primer nivel de experiencia es universal y todo hombre, quiéralo o no, sépalo o no, está llamado a vivirlo de alguna manera en su experiencia de religación.

(48) Cf. HD, 326: “...lo que sucede es que el hombre está fundamentado, y que Dios es la realitas fundamentalis; por lo que la experiencia de Dios por parte del hombre consiste en la experiencia del estar fundamentado fundamentalmente en la realidad de Dios. Haciendo mi ser fundamentalmente es como tengo experiencia de Dios. En la experiencia de Dios lo que hay es la experiencia de la realidad fontanal y fundamentalmente de Dios en la religación como ultimidad, como posibilidad última, y como impelencia suprema".

(49) Ibíd, 327.

(50) Ibíd, 377-379. Zubiri entiende la religación como una marcha en estricto tanteo, en la que cada uno de sus pasos es un verdadero intento de probación que constituye la esencia de lo que se conoce como experiencia.

(51) Ibíd, 328. 
Se trata de aquello que Zubiri ha llamado "experiencia teologal" del hombre, que se expresa a través de las dimensiones: individual, social e histórica. A este primer nivel de la experiencia dedica el primer volumen de su trilogía religiosa, El Hombre y Dios. Al respecto afirma que el modo radical de la experiencia de Dios es la "voluntad de verdad real", es decir, el momento por el cual el hombre es autor de su propio ser personal, el que solo puede llegar a ser buscando apoyo en el poder de lo real que "experiencia" como realidad absolutamente absoluta.

La voluntad se plasma en un proceso intelectivo que ofrece distintas posibilidades de ser a la persona. Cada forma que el hombre adopte es fruto de una opción entendida como apropiación. La opción es la manera de ser libre, es decir, la manera de ser absoluto. A el respecto Zubiri señala: “...la experiencia de Dios de una manera radical y última es la experiencia de mi propia libertad, en tanto que Dios es fundamento de mi propio ser absoluto" (52).

La libertad, en cuanto experiencia radical de Dios, puede tener distintos aspectos. Zubiri reconoce o distingue tres: libertad "de", libertada "para" y libertad "en".

Con la expresión libertad "de" se refiere a la posibilidad de que el hombre pueda ser libre en la medida en que está libre de coacciones externas e internas que lo limiten. Con el término libertad "para" se describe el tener libertad en el sentido de liberación para ser sí mismo. Se es libre para ser una forma de realidad frente a toda otra forma de realidad. Y con la expresión libertad "en" se hace mención al tercer y radical aspecto de la libertad: el ser libre es anterior a todo ejercicio de libertad (de y para). "El hombre es libre en la realidad en cuanto tal. Por ser justamente de aquella condición en virtud de la cual yo soy mío, me pertenezco a mí mismo y no a otra realidad. La libertad en este sentido es o puede ser idéntica a la persona. ...Y en este tercer aspecto de la libertad, es donde está la raíz de mi ser relativamente absoluto y es, por consiguiente, donde está la experiencia del ser libre en la realidad" (53).

Para Zubiri, ser libre es la manera finita y concreta de ser Dios. La experiencia de esta libertad animalmente experienciada es justamente la experiencia de Dios.

\section{c.2.2.2. Experiencia de la gracia:}

Este segundo nivel de la experiencia es el que da origen a la vivencia específicamente religiosa, que en cuanto experiencia es siempre individual, social e histórica. En este sentido, las religiones son siempre una experiencia que se va desarrollando a través del espacio y del tiempo. La tesis de Zubiri al respecto es que Dios es quien se va revelando paulatinamente al hombre y, por lo tanto, la iniciativa no procede del hombre sino de Dios. La historia de las religiones sería el estudio de la revelación o manifestación mundana de Dios, problema estrictamente metafísico filosófico.

Refiriéndose a la experiencia de la gracia o de Dios como gracia, afirma: "El hombre tiene una experiencia de la gracia, aunque no lo sepa, porque nadie hay que esté exento de esta presencia de Dios. Rigurosamente hablando no es una presencia;

(52) Ibíd, 329.

(53) Ibíd, 330. 
es la proyección misma de la vida trinitaria ad extra en lo que consiste, ...la razón formal de la creación del hombre" (54). He aquí la importancia de la historia de las religiones, tema que desarrollará en el segundo volumen de su trilogía religiosa el problema filosófico de la historia de las religiones, al que dedicó largos años de su vida.

\section{c.2.2.3. Experiencia propiamente cristiana: la Encarnación}

Este tercer nivel de la experiencia supone un grado más profundo de experiencia de Dios. Junto con la experiencia de la religación y de la gracia, está la experiencia de la "deiformidad", expresión esencial del cristianismo. Se trata de la experiencia que se hace realidad en la persona de Cristo, en quien Dios asume la forma humana y el hombre se deiformiza, llegando a su máxima expresión la experiencia de Dios por parte del hombre y la del hombre por parte de Dios. Estamos en el culmen de la experiencia teologal que se desarrolla en el mundo como un proceso de deiformación, a través de una experiencia individual, social e histórica.

Para el autor es fundamental captar la manera humana en que Cristo vive su filiación divina, ya que la humanidad fue la experiencia de su filiación divina y no un mero instrumento.

El camino a través del cual el hombre puede acceder al misterio de Cristo es en plenitud la experiencia humana, no en la renuncia a esta experiencia. "Cristo es la experiencia subsistente de Dios. Ciertamente la experiencia subsistente de Dios no es una experiencia al margen de lo que es la experiencia de la vida cotidiana. ...No es experiencia al margen de esto, sino es justamente la manera de experienciar en todo ello la condición divina en que el hombre consiste" (55).

La encarnación le indica al hombre que no debe ocuparse de Dios al margen de sí mismo, de las cosas o del mundo, sino que debe hacerlo ocupándose de las cosas, consigo mismo y las demás personas. Su experiencia de Dios, lejos de alejarlo del mundo lo obliga al mundo. "El hombre tiene que ver en este mundo con todo, hasta con lo más trivial. Pero tiene que ver con todo divinamente. Justo ahí es donde está la experiencia de Dios" (56).

\section{c.2.3. Experiencia de Dios y respuesta humana}

Zubiri es muy consciente de la existencia de distintas y valederas respuestas frente al problema de Dios, que en último término es Dios mismo (57). Lo importante aquí es captar que para el autor todas estas actitudes son respuestas y, por lo tanto, modos de la experiencia de Dios. Ningún hombre podría desentenderse del problema de su fundamento y de su propia constitución, termine este en el reconocimiento de Dios por el creyente, en su negación por el ateo, en la frustración del agnóstico o en la indiferencia del indiferente. Lo fundamental es reconocer que todo

(54) Ibíd, 330-331.

(55) Ibíd, 333.

(56) Loc. cit.

(57) Cf. acápite 1. a. Me limito aquí a esbozar lo fundamental de la reflexión de Zubiri sobre las actitudes que caben frente a la experiencia de Dios. 
hombre está religado al poder de lo real, lo primario no es estar con o sin Dios, sino estar religado al poder de lo real.

En último término, lo importante es que la experiencia de Dios, por parte del hombre, acontece en el propio hacerse persona, en su plenitud personal, la de su vida y de su muerte, y no en la renuncia a ser y a hacerse aquello a lo que se está religado. Y por parte de Dios es entrega, donación de sí mismo, a la experiencia humana para que este alcance su plenitud. Al respecto Zubiri afirma:

"La experiencia de Dios, en consecuencia, a parte Dei, es Dios dándose como absoluto a la experiencia humana; a parte hominis, es haciendo la experiencia de lo absoluto en la constitución de mi persona. El hombre no encuentra a Dios primariamente en la dialéctica de las necesidades y de las indigencias. El hombre encuentra a Dios precisamente en la plenitud de su ser y de su vida. Lo demás es tener un triste concepto de Dios. ...(el hombre) No va por la vía de la indigencia sino de la plenitud, de la plenitud de su ser, en la plenitud de su vida y de su muerte. El hombre no va a Dios en la experiencia individual, social y histórica de su indigencia; esto interviene secundariamente. Va a Dios y debe ir sobre todo en lo que es más plenario, en la plenitud misma de la vida, a saber: en hacerse persona. En el ser personal, en el ser relativamente absoluto de la persona, es donde encuentra a Dios, dándose al hombre en la experiencia suya. Esta donación de Dios es justamente la realidad de la persona. Y esta experiencia humana de lo absoluto es experiencia de esta donación de Dios" (58). (sic)

\section{SÍNTESIS FINAL}

Zubiri utiliza el concepto de religación para referirse a una experiencia humana fundamental, previa a cualquier intento de conceptualización, que expresa la existencia humana como puesto por atrás o desde atrás en la realidad mundana, la única realidad a la que el hombre puede acceder. Realidad que a través del poder de lo real se apodera del hombre, lo religa y lo lanza en una búsqueda de intelección racional de su fundamento en la propia realidad.

El concepto de experiencia que utiliza debe comprenderse en el contexto mayor de lo que entiende por la razón. Su concepto de razón, de carácter incluyente, intenta dar cuenta de la unidad fundamental de las dimensiones que constituyen al hombre y por ello es un concepto amplio y dinámico. La razón es en primer lugar método y consta fundamentalmente de dos pasos: el esbozo y la experiencia. Para el caso de Dios, el esbozo, que es un argumento siempre lógico, tiene directa relación con la religación y consiste en la formulación de un argumento sobre Dios. El segundo elemento, el de la experiencia, es el momento de la probación física de la realidad esbozada, es decir, es el momento de la prueba de verdad real de lo esbozado lógicamente. La posibilidad o imposibilidad de dar el salto desde la religación a la realidad personal de Dios depende de que ambos pasos se realicen adecuadamente. En este contexto, se entiende el concepto de experiencia que utiliza el autor

(58) HD, 344. El paréntesis es mío. 
definido como una prueba, que no tiene carácter de comprobación de ningún tipo, sino de probación física de la realidad, tal como si se tratara de probar manjar.

Para Zubiri el hombre hace la experiencia de religación en la medida en que en su religación está religado precisa y formalmente al poder de lo real. Poder que está en las cosas reales y que a través de ellas y en ellas se manifiesta. Es la experiencia de aprehender en y por la propia realidad lo que es la realidad y el poder de lo real, en cuanto realidad fundamento de la propia realidad desde la que el hombre hace y se hace.

La religación en cuanto momento fundamental de la realidad personal implica que la realidad de Dios, absolutamente absoluta y en esa medida distinta de las cosas reales, y de las realidades personales, está presente en toda realidad personal en cuanto esta se hace y hace. Este carácter trascendente de Dios en las realidades personales se entiende desde su ser realidad fundamento fundamentante de todo lo real. En este sentido, la religación es una presencia de Dios en las cosas precisamente para constituirlas como reales, y específicamente en la persona humana como aquello que está constituyendo su remisión al fundamento de su propia realidad personal en la configuración de sí mismo. Esta relación particular de Dios con el hombre se debe a la propia comprensión de la realidad humana como esencia abierta, es decir, como realidad que se pertenece a sí misma. En la experiencia de lo real el hombre y Dios van radicalmente unidos, cada uno en la realidad de su propia identidad personal, determinados en y por esa experiencia de Dios como lo fundamentante y constitutivo de su realidad personal. En este sentido el hombre es experiencia de Dios y Dios es experiencia del hombre.

Afirmar que el hombre es experiencia de Dios es comprender que en su propia realidad personal el hombre está experienciando la realidad de Dios. No se trata de una experiencia externa a su propia experiencia de realidad personal, sino que en ella y por ella descubrir la presencia de la realidad de Dios que le fundamenta y permite ser real. Dios no es una realidad externa al hombre, ni un objeto con el que este se relaciona, sino una realidad fundamento constitutiva de su realidad experienciada concretamente.

Este ser experiencia de Dios acontece en la estructura fundamental de la naturaleza humana como momento de finitud. El hombre, para Zubiri, es una manera finita de ser Dios. Su experiencia de Dios pasa necesariamente por la asunción en Dios de su propia realidad humana fundamental en su estructura de finitud y no en la postergación o eliminación de su realidad finita. La propia relatividad absoluta del hombre es justamente su experiencia de Dios.

Zubiri distingue en esta experiencia de Dios que el hombre es tres niveles diferentes de experiencia, cada uno de los cuales está cruzado por las dimensiones individual, social e histórica. En primer lugar, reconoce el nivel de la experiencia universal de religación que es común a todo ser humano en la búsqueda de su fundamento y en el ejercicio de su libertad como experiencia radical de Dios. En segundo lugar, concibe la experiencia de la dimensión de la gracia, que es específicamente la vivencia religiosa entendida como la experiencia de la revelación paulatina de Dios al hombre. El último nivel de experiencia que reconoce es el de la experiencia propiamente cristiana, es decir, la experiencia de la Encarnación. Este tercer nivel es el más desarrollado y al que apuntan los dos anteriores, pues es el que 
llevaría al hombre a la experiencia de la deificación dada en Jesucristo, en quien Dios asume la forma humana y el hombre se deiformiza, llegando a su máxima expresión la experiencia de Dios por parte del hombre y del hombre por parte de Dios.

En último término, para Zubiri lo importante es que la experiencia de Dios, por parte del hombre acontece fundamentalmente en el propio hacerse persona y en la plenitud de la persona y en ningún caso en la eliminación o postergación de lo humano frente a lo divino. Dios no es rival del hombre, es su realidad fundamentarte que en donación y entrega de sí mismo posibilita que el hombre se haga real y pleno. En este proceso es preciso reconocer las posibles y variadas respuestas humanas frente al tema de Dios como problema del hombre.

\section{RESUMEN}

El artículo aborda, en un primer acercamiento, el pensamiento de Xavier Zubiri respecto de la experiencia, en cuanto vía de acceso del hombre a Dios, desarrollado especialmente en su trilogía religiosa.

Una clave fundamental de lectura del tema es el concepto de "experiencia de religación" expresado a través de tres problemas nodales: el problema de la realidad, el problema de la inteligencia y el problema de Dios.

Se parte del principio del "poder de la realidad" que se impone al hombre más allá de su voluntad, estableciendo la "experiencia" como "probación física de la realidad", en un sentido diferente al expresado bajo el concepto de empeiría. Siendo Dios el fundamento de todo lo real, el hombre queda religado experiencialmente, físicamente, a esta realidad absoluta, en la medida en que Dios la constituye. Y si el hombre es parte de esa realidad absoluta participa de su esencia y por lo tanto establece una relación con Dios desde su propia plenitud y no desde su finitud como suele pensarse. Esto es lo que hace posible, según el autor, que la experiencia cristiana de la encarnación sea la máxima expresión del acceso humano a Dios.

\section{ABSTRACT}

This article surround, in a first approach, Xavier Zubiri's thought on respect the experience, as a path of access that man have on the way to God, that the author has developed in his religious trilogy.

A foremost clue of lecture on the theme is the concept of "experiencia de religación" (like reliant experience). That appears expressed in three nodal problems: The reality problem, the intelligence problem and the problem of God.

We start from the "power of reality" as a principle that act as an imposition to man beyond his will, establishing the "experience" as a "probación física de la realidad" (like reality verification), in a different sense of empeiria (empirical) concept in traditional metaphysics. Being God the fundament of the hole real, man remains experientially, physically reliant, to this absolute reality, as God constitute it. And if man is part of that absolute reality, he participates of his essence, for what he establish a relation with God from his own plenitude and not from his finitude as it is commonly thought. This makes possible, in the author's believe, that the Christian experience of incarnation becomes the maximum expression of human access to God. 